Revista de Estudios Histórico-Jurídicos

[Sección historia del derecho europeo]

XLI (Valparaíso, Chile, 2019)

[pp. 179 - 205]

\title{
EL SURGIMIENTO DEL JUDICIAL CASE MANAGEMENT: UNA SINTESIS EVOLUTIVA DEL CONTROL JUDICIAL DEL PROCESO CIVIL EN EUROPA
}

[The beginning of the judicial case management: an Evolutionary overview on Judicial control over civil proceedings in europe]

\author{
Ramón García Odgers* \\ Universidad Católica de la Santísima Concepción, Chile
}

\begin{abstract}
RESUMEN
Una perspectiva evolutiva de los principales hitos normativos que demarcan el surgimiento del judicial case management en Europa, permite constatar que el mayor control judicial sobre la litigación civil, como herramienta de eficiencia, constituye una tendencia muy consistente. Las mayores responsabilidades asignadas a los jueces han generado una transformación y ampliación del rol que tradicionalmente se les ha asignado, lo que se justifica en cambios de la filosofía sobre los fines del proceso.
\end{abstract}

\section{Palabras clave}

Judicial case management - proceso civil - evolución histórica.
ABSTRACT

An evolutionary perspective on the main regulatory milestones that determine the beginning of the judicial case management in Europe makes it possible to verify that an increased judicial control over civil litigation, as an efficiency tool, comprises a highly consistent tendency. The main responsibilities assigned to judges have promoted a transformation and growth of the role that has been assigned to them, which is justified through changes in philosophy regarding the aims of the process.

\section{KEYWORDS}

Judicial case management - civil proceedings - historical evolution.

RECibido el 21 de agosto de 2018 y ACEPTADO el 30 de abril de 2019

\section{INTRODUCCIÓN}

El control judicial sobre el desarrollo de la litigación civil en Europa, se configura actualmente bajo el concepto de judicial case management, que comienza, aun cuando la terminología como tal aún no había sido inventada, en la Europa del siglo XIX ${ }^{1}$. Si bien su versión más reciente e institucionalizada corresponde a las Civil Procedure Rules del Reino Unido, sus lógicas y algunas herramientas

\footnotetext{
*Abogado, Doctor en Derecho por la Pontificia Universidad Católica de Valparaíso; Profesor
} 
también se manifiestan en sistemas procesales de tradición continental ${ }^{2}$, donde ya se reconocía un precedente relevante en la Ordenanza Procesal Civil Austriaca ${ }^{3}$.

Para los efectos de esta introducción, consideraremos que la esencia del case management"es que el sistema judicial en su conjunto y, los tribunales en los casos individuales, regulan el contenido y el progreso de la litigación"4.

Desde el punto de vista histórico, este case management aparece como el producto de una larga y compleja evolución, que puede sintetizarse como la sustitución de las concepciones ideológicas y teóricas individualistas sobre la función y operatoria de la justicia civil ${ }^{5}$.

En efecto, la tradición procesal europea que nació con la revolución francesa con una actitud de laissez faire y un enérgico rechazo a la discreción judicial, fue transformada paulatinamente 6 . Un hito inicial, en esta evolución, lo constituyó el código austriaco que entró en vigor en 1898. Las características de este Código se corresponden con la función social del proceso, según la cual, los jueces debían participar activamente en la gestión de los casos ${ }^{7}$. Este enfoque tenía como objetivo permitir que las demandas procedieran de manera eficiente, expedita y económica para el juicio, y la sentencia sobre sus méritos sustantivos ${ }^{8}$.

Casi cien años después, en 1999, Inglaterra incorporó en las Civil Procedure Rules, el judicial case management como herramienta central para el cumplimiento de diversos objetivos, y especialmente, del denominado overriding objective (objetivo predominante $)^{9}$. Este último busca asegurar un tratamiento justo, económico y

de Derecho Procesal y Litigación de la Universidad Católica de la Santísima Concepción. Correo electrónico: rgarcia@ucsc.cl. Dirección postal: Lincoyan 255, Concepción, Chile.

${ }^{1}$ Van Rhee, C.H., Introduction, en Van Rhee, C. H. (ed.), Judicial Case Management and Efficiency in Civil Litigation (Intersentia Antwerp - Oxford - Portland, 2008), p. 2. En relación al procedimiento francés, Cadiet indica que las modificaciones al Código de 1976, han reforzado el principio de cooperación entre el juez y las partes, que manifiesta la preocupación acumulada por la racionalización del proceso que se expresa hoy a través del desarrollo de lo que se denomina judiciary management o judicial case management (gestión judicial de casos). CADIET, Löic, Introduction to french civil justice system and civil procedural law, en Ritsumeikan Law Review, 28 (2011), pp. 366 y 367.

${ }^{2}$ Para el caso Europeo, Cfr. CEPEJ Report on "European judicial systems - Edition 2014 (2012 data): efficiency and quality of justice", p. 480, disponible en World Wide Web: http:/ / www.coe. int/t/dghl/cooperation/cepej/evaluation/2014/Rapport_2014_en.pdf..

${ }^{3}$ VAN RHEE, Introduction, cit. (n. 1), pp. 2 ss.

${ }^{4}$ Andrews, Neil, English civil procedure. Fundamentals of the new civil justice system (New York, Oxford University Press, 2003), p. 337.

${ }^{5}$ Se trata del declive progresivo del concepto bipartidista de laisser-faire de la justicia civil. Cappellettr, Mauro, Vindicating the public interest through the courts: A comparativist's contribution, en Buffalo Law Review, 25 (1975-1976), p. 689.

${ }^{6}$ Marcus, Richard, Misgivings about american exceptionalism: Court access as a zero-sum game, en Uzelac, Alan - VAn Rhee, C. H. (eds.), Revisiting procedural buman rights: Fundamentals of civil procedure and the changing face of civil justice (Cambridge, Intersentia Metro, 2017), p. 55.

${ }^{7}$ Van Rhee, C. H.; The development of civil procedural law in twentieth-century Europe, en Russian Yearbook on Civil Procedure \& Arbitration, 7-8 (2011), pp. 11-25.

${ }^{8}$ SorabjI, John, Managing claims (Tianjin Conference, IAPL, 2017), p. 2.

${ }^{9}$ Andrews, Neil, Andrews On Civil Processes (Intersentia Cambridge, Antwerp Portland, 2013), I, p. 196. 
expedito para todos los casos, bajo un enfoque de estricta proporcionalidad ${ }^{10}$. Tras este ideal se encuentra la noción de justicia distributiva, que exige que los limitados recursos de la justicia deban ser justa y equitativamente distribuidos ${ }^{11}$. Esto implica que el interés del caso individual debe equilibrarse con el interés público de asegurar a todos los litigantes una parte efectiva y equitativa de los recursos del sistema ${ }^{12}$.

Desde esta vitrina, y bajo la configuración más elaborada de las Civil Procedure Rules (CPR) que siguieron al Informe de Lord Woolf ${ }^{13}$, el judicial case management ha recibido especial atención de la doctrina procesal comparada ${ }^{14}$, generando nuevas perspectivas de análisis académico, propuestas de reformas o derechamente la recepción de este instituto, en países de todo el mundo ${ }^{15}$. Además, al reducir su carácter adversarial, y aumentar el control de los jueces sobre la litigación el proceso civil inglés se ha acercado al de sus contrapartes continentales ${ }^{16}$.

Una observación general de los aspectos involucrados en esta evolución, permite constatar como trasfondo, la existencia de una tendencia consistente hacia un control del sistema judicial sobre la litigación como mecanismo de eficiencia, desplazando el tradicional dominio de las partes sobre el proceso de adjudicación ${ }^{17}$. Para establecer un hilo conductor de este trabajo y acotar sus alcances, nos enfocaremos en tres influyentes codificaciones procesales europeas. El contexto histórico en que surgieron, los principios más relevantes que las inspiraron, la configuración del rol del juez, y su influencia en el contexto europeo nos servirán como ejes centrales de este desarrollo ${ }^{18}$.

De esta forma, este trabajo comienza ofreciendo (I) una síntesis de la configuración del proceso civil en Europa previo a las codificaciones europeas, la que

${ }^{10}$ Cfr. artículo 1.1, de las Civil Procedure Rules, disponibles en World Wide Web: http:// www.justice.gov.uk/courts/procedure-rules/civil (consulta, 22 de marzo de 2017).

${ }^{11}$ ZuCKERMAN, Adrian A.S., Justice in crisis; comparative dimensions of civil procedure, en ZuCKERMAN, Adrian A.S. (ed.), Civil justice in crisis, comparative perspectives of civil procedure (Oxford, Oxford University Press, 1999), p. 48.

${ }^{12}$ SORABJI, John, English civil justice after Woolf and Jackson reform: A critical analysis (London, Cambridge University Press, 2014), p. 233.

${ }^{13}$ Jolowicz, J. A., On civil procedure (First ed., Cambridge University Press, 2000), p. 52.

${ }^{14}$ Andrews, Neil, A new civil procedural code for England: Party control "going, going, gone", en Civil Justice Quarterly, 19 (2000), p. 19.

${ }^{15}$ Van Rhee, C. H., Judicial Case Management and Efficiency in Civil Litigation (Intersentia Antwerp - Oxford - Portland, 2008).

${ }^{16}$ STURnER, Rolf, Some European remarks on a new joint project of the American Law Institute and UNIDROIT, en The International Lanyer, 34/3 (2000), p. 1076.

${ }^{17}$ Cappellettr, Mauro, Who watches the watchmen? A comparative study on judicial responsibility, en American Journal of Comparative Law, 31/1 (1983), pp. 6-9.

${ }^{18} \mathrm{La}$ identificación de estas codificaciones, como hitos generales del desarrollo procesal civil europeo, se observa inicialmente en la obra de Cappelleti y Garth. Véase CAPpelLETTI, Mauro, GARTH, Bryant G., International encyclopedia of comparative law XVI (Dordrecht, Boston, Lancaster, J.C.B Mohr (Paul Siebeck), Tubingen y Martinus Nijhoff Publishers, 1987), I, senda continuada en la obra del professor Van Rhee. Véase, entre otros, Van Rhee, C.H., European Traditions in civil procedure, (Intersentia Antwerpen - Oxford, 2005) y VAN RHEE, C.H., Judicial Case Management and Efficiency in Civil Litigation (Intersentia Antwerp - Oxford - Portland, 2008). 
corresponde, en términos generales, al modelo de procedimiento del ius communne. A continuación, centramos el trabajo en los principales códigos europeos que marcan un alejamiento del paradigma anterior, comenzando con (II) el Código de Procedimiento Civil francés de 1806, dominado por el liberalismo del siglo XIX, con un enfoque privado y fuertemente centrado en la autonomía de las partes. Luego, se revisa (III) el Código Austriaco de fines del siglo XIX, que marca una importante inflexión en la tendencia predominante, avanzando hacia una desprivatización del modelo procesal. Asociado a la oralidad se incorpora la idea de un juez con amplias facultades tendientes a equilibrar los intereses privados de los litigantes con los alcances sociales del proceso, introduciendo en la arquitectura del procedimiento civil, la consideración de factores ajenos al caso individual. La última etapa de esta síntesis se ocupa de (IV) las Civil Procedure Rules (CPR) de Inglaterra y Gales, que junto con reducir los poderes de las partes en la conducción de la litigación, incrementó e institucionalizó los poderes de case management de los jueces para el cumplimiento de los objetivos del sistema de justicia civil. Al finalizar, se expondrán algunas reflexiones a modo de conclusión.

El objetivo general de este trabajo se orienta a contribuir al entendimiento del judicial case management, considerado actualmente como uno de los tópicos más relevantes para la teoría procesal civil, posicionándose fuertemente en el universo de ideas y categorías procesales, fundamentalmente en la doctrina y derecho comparado ${ }^{19}$.

La síntesis histórica que presentamos a continuación, resulta útil para comprender que el case management se encuentra inserto en una de las tendencias más relevantes del derecho procesal contemporáneo. Este análisis, que incluye referencias al derecho histórico, permite, además, identificar los paradigmas que contribuyeron a su origen. Finalmente, también resulta útil para examinar y reconocer cómo se inserta nuestro propio sistema de justicia en esta evolución, reconociendo factores presentes como, por ejemplo, la incorporación de la oralidad y una mayor preocupación por los alcances sociales y económicos de los procesos.

\section{LA REACCIÓN CONTRA EL PROCEDIMIENTO DEL IUS COMMUNE}

\footnotetext{
${ }^{19}$ En el Congreso Mundial de Derecho Judicial de Coimbra, en 1991, se concluyó la necesidad que el juez se convierta en manager. BERIZONCE, Roberto Omar, Recientes tendencias en la posición del juez: Relación general, en BERIzOnCE, Roberto Omar (coord.), Eljuezy la magistratura (Tendencias en los albores del siglo XXI) (Santa Fe, Rubinzal Culzoni Editores, 1999), p. 45. En la reunión de la Asociación Internacional de Derecho Procesal, desarrollada el 2009 en Toronto, se asumió el case management como una redefinición del rol del Juez. OTEIZA, Eduardo, Civil procedure reforms in Latin America: The role of the judge and the parties in seeking a fair solution, en The Supreme Court Review, 49 (2010), pp. 227 ss. El 2017, en Tianjin, China, el Coloquio de la Asociación Internacional de Derecho Procesal, versó sobre el case management desde la perspectiva comparada. Consúltese: sitio web de la Asociación Internacional de Derecho Procesal, en http://www.iaplaw.org.
} 
En la tradición del civil law el dominio del viejo y escrito procedimiento del ius commune, se quebró sólo después de una larga lucha, que culminó con la adopción de los códigos de los siglos XVIII y XIX ${ }^{20}$.

El procedimiento de ius commune estaba fundado sobre los principios de escrituración, de mediación y de completa inactividad y neutralidad del juez ${ }^{21}$, al cual debía sustraerse toda dirección del proceso ${ }^{22}$. Este procedimiento constituyó el paradigma prevalente desde la edad media, lo que se explica, en parte, por la falta de un sistema judicial respetado y poderoso ${ }^{23}$. Esto resulta evidente desde la decretal del papa Inocencio II del año 1216, que declaró que todo acto del procedimiento debía ser escrito y el juez sólo podría decidir el asunto en base a lo escrito, para proteger a las partes contra las "falsas declaraciones de jueces injustos" 24 . En consecuencia, el desarrollo del litigio se entregó a las partes, con el fin de protegerlas de la arbitrariedad de los jueces ${ }^{25}$.

Contribuyó a reforzar esta concepción, el principio de control de parte sobre el objeto del proceso (Dispositionsmaxime), cuyo entendimiento tradicional considera que el juez es relativamente pasivo y son las partes las que dominan el litigio ${ }^{26}$. De este modo, el proceso civil fue concebido como un instrumento para la resolución de controversias, donde el control del inicio, progreso y la finalización del procedimiento se entregó a los litigantes ${ }^{27}$, considerados los domini litis $^{28}$.

En suma, el menor rol de los jueces en el desarrollo de la litigación fue una característica del procedimiento civil del ius communne ${ }^{29}$, el que fue considerado un asunto privado, lo que implicaba no sólo el control de los derechos sustantivos en disputa, sino que, también, la dominación de las partes sobre el desarrollo del procedimiento $^{30}$.

Frente a este paradigma, se generó el movimiento de reforma, en el cual, la

${ }^{20}$ Homburger, Adolf, Functions of orality in Austrian and American civil procedure, en Buffalo Law Review, 20 (1970), p. 11.

${ }^{21}$ Cappellettr, Mauro, Social and political aspects of civil procedure: Reforms and trends in western and eastern Europe, en Michigan Law Review, 69/5 (1971), pp. 847-850.

${ }^{22}$ LangBeIn, John, Bifurcation and the bench: The influence of the jury on english conceptions of the judiciary, en BRAND, Paul - GETZLER, Joshua (eds.), Judge and judging in the history of common law and civil law: From antiquity to modern times (Cambridge University Press, 2012), pp. 68 ss.

${ }^{23}$ Cappelletti, Mauro, Garth, Bryant G., International encyclopedia, cit (n.18), p. 23.

${ }^{24}$ Ibíd.

${ }^{25}$ Frodu, Susanne, The heritage of the Franz Klein reform of Austrian civil procedure in 1895-1896, en Civil Justice Quarterly, 31/1 (2012), p. 45.

${ }^{26}$ VAn RheE, C. H., Towards a procedural ius commune?, en Smits, J. - LubBE, G. (eds.), Remedies in Zuid-Afrika en Europa (Antwerpen, 2003), p. 231.

${ }^{27}$ Damaska, Mirjan, The common law / civil law divide: Residual truth of a misleading distinction, en Chase, Oscar G. - Walker, Janet (eds.), Common Law, Civil Law and the Future of Categories (Markham, LexisNexis, 2010), pp. 3 ss.

${ }^{28}$ Ibíd, p. 4.

${ }^{29}$ Para una descripción de la historia y características del procedimiento del ius commune, véase: Engelmann, Arthur et al, History of continental civil (trad. de Robert Wyness Millar, Boston, Little Brown \& Co., 1927), pp. 455-492.

${ }^{30}$ Cappelletti, Mauro, Garth, Bryant G., cit. (n. 18), p. 23. 
oralidad fue un tema central, ya que representó una nueva doctrina procesal ${ }^{31}$. Sin embargo, su denominación como o r a li d a d fue engañoso, ya que significaba mucho más que eso ${ }^{32}$. Implicaba una reacción en contra, y un radical rompimiento con todas las características del procedimiento de jus commune ${ }^{33}$. De acuerdo a esto, además de una revalorización del elemento oral, sus principales ideales fueron, la in mediatez; una libre o crític a evaluación de pruebas; la concentración del caso en una sola audiencia, preparado en una etapa preliminar. Por último, y como consecuencia de los tres primeros ideales, un desarrollo más rápido del litigio ${ }^{34}$.

Un juicio oral necesita un procedimiento concentrado para la evaluación de la prueba de los hechos. La concentración, requiere, a su turno, de un firme control sobre la preparación y desarrollo del litigio. Cuando se removió la barrera de la escrituración entre el juez y las partes y su litigio, el juez ya no podía mantenerse como un espectador, sino que se convirtió en un activo participante, dirigiendo y controlando el desarrollo del litigio ${ }^{35}$.

\section{El CODE DE PROCÉDURE CIVILE}

\section{Antecedentes}

Según Van Rhee, la historiografía del proceso civil del siglo XX en Europa, desde la perspectiva del case management y la eficiencia, comienza en el siglo 19 con el Code de procédure civile francés adoptado en $1806^{36}$. Durante el período de la codificación, fue este cuerpo normativo el que introdujo alguna de las características básicas del procedimiento del ius commune ${ }^{37}$, aunque con ciertas manifestaciones de la oralidad y del proceso adversarial ${ }^{38}$, que operaron de un modo imperfecto ${ }^{39}$.

En efecto, la Revolución Francesa, además de otras innovaciones en el terreno judicial, generó una ruptura con el pasado ${ }^{40}$. Pese a que fue parcial e incompleta, es comprensible que el movimiento intelectual favorable a la reforma -que se manifestó en otros países de Europa y que tuvo como su impulso el principio de oralidad y de publicidad en el proceso en la primera mitad del siglo XIX-

\footnotetext{
${ }^{31}$ Homburger, Adolf, cit. (n. 20), p. 11.

${ }^{32}$ Cappelletti, Mauro, La oralidad y las pruebas en el proceso civil (trad. de Santiago Santis Melendo, Buenos Aires, Ediciones Jurídicas Europa - América S.A., 1972), p. 45.

${ }^{33}$ Cappellettr, Mauro, Social and political, cit. (n. 21), pp. 853-854.

${ }^{34}$ Ibíd.

${ }^{35}$ Cappelletti, Mauro, Garth, Bryant G., cit. (n. 18), p. 24.

${ }^{36}$ VAN RheE, C. H., European traditions, cit. (n18), pp. 3-4.

${ }^{37}$ Van Rhee, C. H., Verkerk, R., Civil procedure, en Smits, Jan M. (ed.), Elgar encyclopedia of comparative law (Second ed., Edward Elgar Publishing Limited, 2012), pp. 123 ss.

${ }^{38}$ CADIET, Löic, Sources and destiny of french civil procedure in a globalized world, en PICKER, Coli B. - SeIdman, Guy I. (eds.), The dynamism of civil procedure. Global trends and developments, en Ius Gentium: Comparative Perspective on Law and Justice, 48 (Springer International Publishing, 2016), p. 66.

${ }^{39} \mathrm{El}$ modelo francés tenía un significado predominante de la oratoria forense, imperfecta e insuficiente. Cfr. CAppellettr, Mauro, La oralidad, cit. (n. 32), p. 47.

${ }^{40}$ Para una descripción de las características de los tribunales en el ancient regime, véase: VAN Caenegem, R.C., An historical introduction to private law (trad. de Johnston, D.E.L., Cambridge University, 1992), pp. 100 ss.
} 
considerara a Francia como la vanguardia, y en muchos aspectos, como modelo de un sistema o r a l; entendiendo como tal, un proceso distinto del c o m ú $\mathrm{n}^{41}$.

Con todo, mucho antes de la Revolución ya se habían hecho esfuerzos para remediar los problemas del procedimiento civil, en especial, para hacerlo más simple, rápido y menos costoso ${ }^{42}$. Nos referimos principalmente a la Ordonnance civile pour la reformation de la justice (1667), conocida como el Código de Louis, la Ordonnance sur le commerce (1673), conocido como el Code Marchand o Code Savary, y la Ordonnance sur le commerce des mer u Ordonnance de la Marine (1681), las que reconocieron la importancia de una buena administración de justicia para el desarrollo del comercio y la industria, y fueron consideradas fundamentales en la claridad y seguridad jurídica ${ }^{43}$.

De igual modo, estas preocupaciones por la economía se expresaron en la exposición de motivos del code du procedure civile ${ }^{44}$. Así, Treilhard, durante la presentación del proyecto, señaló que "todo estaba previsto para hacer el procedimiento más simple, más corto y más económico, las formalidades inútiles abolidas y los procedimientos superfluos abolidos" ${ }^{45}$.

El Código francés de $1806^{46}$, fue producto de un extenso debate ${ }^{47}$, y una mezcla o síntesis del acervo revolucionario y del tradicionalismo reaccionario ${ }^{48}$. Su fuente y referencia principal, fue el sistema tradicional heredado del Antiguo Régimen $^{49}$, con ciertos matices ${ }^{50}$. En este sentido, aparece como la expresión de una sociedad y de un régimen político que requería poner los años revolucionarios detrás de ellos, pero sin poner el reloj totalmente de vuelta a la era del Ancien Régime ${ }^{51}$.

En Francia, aun antes de la Revolución, el proceso estaba modelado, más bien, sobre el esquema del proceso común sumario que sobre el esquema, más

${ }^{41}$ Cappelletti, Mauro, La oralidad, cit. (n. 32), p. 46.

${ }^{42}$ CADIET, Löic, Case management judiciaire et déformalisation de la procédure, en Revue Française d'Administration Publique, 125/1 (2008), p. 137.

${ }^{43}$ VAN CAenegem, cit. (n. 40), pp. 91 ss.

${ }^{44}$ CADIET, Löic, cit. (n. 42), p. 137.

${ }^{45}$ CADIET, Loïc, La justicia civil francesa entre eficiencia y garantias, en Civil Procedure Review, $3 / 4$ (2013), p. 28.

${ }^{46}$ Una descripción de la estructura del Code de Procedure Civile, vease en EngELmann, Arthur et al., cit. (n. 29), pp. 748-782.

${ }^{47}$ Soulez Lariviere, Daniel, Overview of the problems of french civil procedure, en The American Journal of Comparative Law, 45 (1997), p. 737.

${ }^{48}$ WijfFels, Alan, The code de procédure civile (1806) in France, Belgium and the Netherlands, en VAn Rhee C. H.; Heirbaut, D.; STORMe, M. (eds.), The french code of civil procedure (1806) after 200 years: The civil procedure tradition in France and abroad (Kluwer, 2008), p. 5.

${ }^{49}$ BAKER, Wallace R., French judgments subject to immediate appeal, en Law and Contemporary Problems, $47 / 3$ (1984), p. 21.

${ }^{50}$ CADiET, Löic, The international sources of french civil procedure, en DEGuchi, Masahisa -STORME, Marcel (eds.), The reception and transmission of civil procedural law in the global society. Legislative and legal educational assistance to other countries in procedural law (AIPL, Maklu Antwerpen - Apeldoorn, Maklu Publishers, 2008), p. 263.

${ }^{51}$ WijfFels, Alan, French civil procedure (1806-1975), en VAN RHEE, C. H. (ed.), Europeans traditions in civil procedure (Intersentia Antwerpen - Oxford, 2005), p. 25. 
pesado y formal, del proceso común ordinario, y además, existía con cierta importancia práctica, vinculada a la importancia de la abogacía, una fase de debate oral, después de los escritos preparatorios y de la práctica de las pruebas. De ahí que las reformas de la legislación revolucionaria y posrevolucionaria se insertaron sobre una práctica más flexible que la predominante, por ejemplo, en las regiones del centro de Europa ${ }^{52}$.

\section{La filosofía del Code de Procedure Civile. El liberalismo y el control de las partes sobre} el proceso

El Código de 1806 puso el juicio en manos de las partes $^{53}$, lo que significaba su dominio sobre las reglas que establecen los plazos, formas y modalidades de las actividades relacionadas al proceso ${ }^{54}$. Como muchas doctrinas del siglo 19, impresas en el Código Civil, el procedimiento civil se basó en la ficción que las partes estaban en posición para hacer elecciones racionales y asumir el manejo de sus asuntos, incluida la litigación ${ }^{55}$.

En este sentido, el Code de Procedure Civile reflejó la actitud liberal del siglo $\mathrm{XIX}^{56}$, con su énfasis en la responsabilidad individual de los ciudadanos, considerados como hombres razonables que litigarían en una posición de igualdad ${ }^{57}$. Como resultado de ello, el rol de los jueces fue reducido a una suerte de notario o árbitro, un testigo de los actos procesales de las partes ${ }^{58}$, sin mayor poder para intervenir en el proceso en pos de la eficiencia y para prevenir el retraso excesivo ${ }^{59}$.

Si bien existió una intención declarada de reducción de costos y demoras ${ }^{60}$-argumento recurrente en los travaux préparatoires- los legisladores no la manifestaron en concreto en las normas del Código ${ }^{61}$. Sobre las disfuncionales características de la litigación (costos, demoras, tecnicismos, la vulnerabilidad a la chicanería y estrategias de mala fe), prevaleció el enfoque que los abogados eran un grupo responsable y profesionalmente ético, y por ende, fue considerado más efectivo y justo, dejarles a ellos la conducción del procedimiento, con el juez supervisando como un árbitro pasivo ${ }^{62}$.

La filosofía detrás de este enfoque, fue la desconfianza en los jueces, cuyos poderes debían ser suprimidos, para proteger los derechos de las partes ${ }^{63}$. Así, aunque

${ }^{52}$ Cappelletti, Mauro, La oralidad, cit. (n. 32), p. 47.

${ }^{53}$ CADIET, Löic, Introduction to French, cit. (n.1), p. 350.

${ }^{54}$ CARPI, Federico, Le riforme del processo civile in Italia verso il XXI secolo, en Rivista Trimestrale di Diritto e Procedura Civile (Milano, Giuffré Editore, 2000), p. 106.

${ }^{55}$ WijfFels, Alan, France: Powers of the judge, en VAN RHEE, C. H. (ed.), Europeans traditions in civil procedure (Intersentia Antwerpen - Oxford, 2005), pp. 269-270.

${ }^{56}$ Cfr. CADiET, Löic, Introduction to french, cit. (n. 1), pp. 382-383.

${ }^{57}$ Van Rhee, C. H., European traditions, cit. (n. 18), p. 6.

${ }^{58}$ Allemeersch, B., The belgian perspective on case management in civil litigation, en Van RheE, C. H. (ed.), Judicial case management and efficiency in civil litigation (Intersentia Antwerp - Oxford Portland, 2008), p. 81.

${ }^{59}$ VAN RheE, C. H., European traditions, cit. (n. 36), p. 6.

${ }^{60}$ WijfFels, Alan, The code, cit. (n. 48), p. 18.

${ }^{61}$ Ibíd., pp. 18-19.

${ }^{62}$ WijfFELS, Alan, France, cit. (n. 55), p. 270.

${ }^{63}$ Allemeersch, B., cit. (n. 58), p. 81. 
el Código francés de 1806 preveía cierta intervención del juez en la preparación del proceso o mise en état, no tuvo operatividad práctica ${ }^{64}$. Las partes controlaron el procedimiento, y el rol del tribunal consistió sólo en decidir los casos ${ }^{65}$.

\section{Influencia del Código francés de 1806 en Europa}

La imagen del juez pasivo del Código francés dejaría sus marcas sobre el siglo 19 en el proceso civil de casi todos los estados europeos ${ }^{66}$.

En efecto, el modelo francés fue muy influyente, modelando el derecho procesal en diversos países de Europa ${ }^{67}$. Algunos de estos países o territorios habían sido anexados, o se habían vuelto dependientes del Imperio Francés antes de la era Napoleónica y durante la misma ${ }^{68}$, como Bélgica y Holanda ${ }^{69}$. También fue implementado en el Reino de Wesfalia y en el Gran Ducado de Berg ${ }^{70}$.

\section{La Ordenanza Procesal Austriaca: El surgimiento del judicial case MANAGEMENT}

1. El quiebre con el sistema romano canónico. La oralidad y los nuevos principios inspiradores del proceso civil

Tanto dentro como fuera de Europa, el austriaco Zivilprozessordnung con su juez activo asumió la tarea de constituirse en el ejemplo, por sobre el del código francés y de punto de partida del case management y de la consideración de la eficiencia del proceso civil en el siglo $\mathrm{XX}^{71}$.

En efecto, con la Ordenanza Procesal Civil Austriaca de 1895, el procedimiento civil modificó su carácter predominantemente privado tomando una connotación pública, llamada Sozialfunktion del proceso civil, según el cual el procedimiento sirve al bien común ${ }^{72}$.

Este código fue producto de los cambios sociales ocurridos por la revolución industrial, y de demandas articuladas sobre la oralidad, la inmediación y la libre

${ }^{64}$ NgwasiRi, C. N., The role of the judge in french civil proceedings, en Civil Justice Quarterly, 9 (1990), p. 168.

${ }^{65}$ Jolowicz, J. A., Civil litigation: What's it for?, en Cambridge Law Journal, 67/3 (2008), p. 513.

${ }^{66}$ Van Rhee, C. H., The development, cit. (n. 7), p. 11.

${ }^{67}$ VAn RheE, C. H., The influence of the french code de procédure civile (1806) in 19th century Europe, en CAdiet, L. - Canivet, G. (eds.), De la commémoration d'un code à l'autre: 200 ans de procédure civile en France (Paris, LexisNexis/Litec, 2006), pp. 129-165.

${ }^{68}$ PRÜTTING, Hans, International sources of german civil procedure, en DEGUCHI, Masahisa - STORME, Marcel (eds.), The reception and transmission of civil procedural law in the global society. Legislative and legal educational assistance to other countries in procedural law (International Association of Prodedural Law AIPL, Maklu Antwerpen - Apeldoorn, Maklu Publishers, 2008), p. 251.

${ }^{69}$ WijfFels, Alan, The code, cit. (n. 48), pp. 51 ss.

${ }^{70}$ VAn Rhee, C. H.; European traditions, cit. (n. 36), pp. 7 ss.

${ }^{71}$ Van Rhee, C. H.; The development, cit. (n. 7), p. 11. En Europa, la transición hacia un modelo procesal con mayor intervención del juez se desarrolló de la mano de la oralidad en conjunción con el fenómeno de publicitación del proceso civil. CAPPELLETTI, Mauro, El proceso civil en el derecho comparado. Las grandes tendencias evolutivas (trad. de Santiago Sentis Melendo, $1^{a}$ edición, Buenos Aires, Ediciones Jurídicas Europa- América S.A., 1973), pp. 135 ss.

${ }^{72}$ VAn RheE, C.H., Introduction, cit. (n. 1), p.3. 
evaluación de la prueba ${ }^{73}$. Su redactor Franz Klein, se apartó del enfoque liberal, percibiendo la litigación como un fenómeno social negativo y a la administración de justicia como un recurso indispensable para el bienestar ${ }^{74}$. Su principal objetivo fue crear normas procesales que generaran una litigación rápida y económica, que llevara a una sentencia basada en los hechos, tal como ellos habían ocurrido, entregando al juez un rol activo en la conducción del proceso ${ }^{75}$. Para Klein la ley no iba a ser un obstáculo a la justicia y paz social, sino que iba a ser un instrumento que, por medio de ilustrados coloquios llevados a cabo entre el juez y las partes, traería el estado de bienestar ${ }^{76}$.

En suma, desde el punto de vista de su origen, el case management en Europa tiene su raíz en este modelo de juez activo de la Ordenanza Procesal Civil Austriaca, cuyas características se corresponden con la función social del proceso ${ }^{77}$.

Antes de ello, predominaban las características del procedimiento del ius commune.

En efecto, hasta antes de 1898 en Austria se aplicaba el Allgemeine Gerichtsordnung de 1781, caracterizado por ser escrito, secreto, y por la dominación de las partes $^{78}$. Si bien, la Constitución de 1867 ya había declarado que el procedimiento debía ser oral, este mandato constitucional no se llevó a cabo plenamente antes del Código de Klein ${ }^{79}$. Así, por ejemplo, la Ordenanza Judicial General, de 1 de mayo de 1781, consagró un procedimiento escrito ${ }^{80}$.

Esta situación cambió en 1873 para las pequeñas causas, con la implementación del Bagatellverfahren, la que se caracterizó por la oralidad, la inmediación y la libre valoración de las pruebas ${ }^{81}$. Sin embargo, fue en el nuevo Código que se exigió que las alegaciones, el interrogatorio de las partes, la prueba de todos los temas debatidos, e incluso, la sentencia, debían sujetarse al requisito de la oralidad ${ }^{82}$. La reforma se centró en el principio de inmediación (Unmittelbarkeit), que significa que el juez que decide el caso, igualmente debe escuchar la prueba, rechazando el

\footnotetext{
${ }^{73}$ Oberhammer, P. - Domej, T., Improving the efficiency of civil justice: Some remarks from an Austrian perspective, en VAN RHEe, C.H. - UzelaC, Alan (eds.), Civil justice between efficiency and quality: From ius commune to the Cepej (Intesentia Metro, 2008), p. 63.

${ }^{74}$ WaLl, Andrea, Austria \& Germany: A history of successful reforms, en VAN RHEE, C.H. - Yulin, $\mathrm{Fu}$ (eds.), Civil litigation in China and Europe: Essays on the role of the judge and the parties (Dordrecht, Springer, 2014), p. 145.

${ }^{75}$ Van Rhee, C. H., The development, cit. (n. 7), p. 13.

${ }^{76}$ GARTH, Bryan, Franz Klein, Mauro Cappelletti, and the mission of comparative procedural scholars: opening lecture for Intemational Association of Procedural Law, en Revista de Derecho de la Pontificia Universidad Católica del Perú, 52 (1999), p. 556.

${ }^{77}$ VAn RheE, C. H., The development, cit. (n. 7), p. 11-25.

${ }^{78}$ VAN RHEE, C. H.; The development of civil procedural law in twentieth century Europe: From party autonomy to judicial case management and efficiency, en VAN RHEE, C. H. (ed.), Judicial case management and efficiency in civil litigation (Intersentia Antwerp - Oxford - Portland, 2008), p. 12. Y gobernado por las ideas del liberalismo. WALl, Andrea, Austria \& Germany: A bistory, cit. (n. 74), p. 145

${ }^{79}$ LenHoff, Arthur, The law of evidence: A comparative study based essentially on Austrian and New York law, en The American Journal of Comparative Law, 3/3 (1954), p. 316.

${ }^{80}$ Engelmann, Arthur et al, cit. (n. 29), pp. 629-630.

${ }^{81}$ VAn Rhee, C. H.; The development, cit. (n. 78), p. 11.

${ }^{82}$ LenHOFF, Arthur, cit. (n. 79), p. 316.
} 
método por el cual un tribunal tomaría su decisión sobre la base de un informe efectuado por un juez d e l e ga d ${ }^{83}$.

La principal idea de Klein fue organizar el procedimiento civil de un modo que permitiera a los tribunales establecer la verdad de los hechos. En su visión, la sociedad era mejor servida si el derecho sustantivo recibía aplicación, lo que requería establecer la verdad de los hechos ${ }^{84}$. Según esto, el objeto y sentido del proceso se derivan del derecho material, y para hacer este instrumento efectivo, se debía "liberar la fuerza controlada de los jueces y situarla, al igual que la del resto de los poderes estatales, al servicio del derecho, del bien común y de la paz social" ${ }^{15}$.

Por esta razón, Klein tuvo una disposición desfavorable hacia la Verbandlungsmaxime, esto es, el principio de control de las partes sobre las alegaciones y prueba, ya que permitía que intentaran obtener una sentencia incorrecta. Así, el rol activo del juez se estableció para promover la búsqueda de la verdad sustantiva, que podía ser alcanzada por medio de la formulación preguntas de oficio, o por la recolección de pruebas de propia iniciativa, lo que, en la práctica, dependía de la buena voluntad y destreza del juez ${ }^{86}$.

\section{La función social del proceso: La preocupación por los factores sociales y la economía} del proceso

La función social del proceso civil pone a la litigación en un marco mucho más amplio, ya que atiende a dos elementos constitutivos, a saber, que el hecho que resolver disputas específicas no es el único propósito del proceso civil, sino que, también sirve al bien común y que la litigación civil debe ser vista desde un ángulo económico ${ }^{87}$.

En efecto, el pensamiento procesal de Klein entendía cada disputa legal como un mal social, que afectaba negativamente la economía ${ }^{88}$. El proceso, entonces, cumple el rol de un médico que diagnóstica una enfermedad y prescribe la cura, como un servicio social para el bienestar de la sociedad y del litigante individual ${ }^{89}$. El juez sería responsable por un juicio justo, eficiente y socialmente bien balanceado $^{90}$. Los procedimientos debían ser simples, comprensibles y predecibles, y los gastos de personal, dinero y tiempo debían mantenerse en el mínimo ${ }^{91}$.

En este contexto, el proceso no podía seguir siendo considerado como una

${ }^{83}$ Ibíd., p. 318.

${ }^{84}$ GRISs, Irmgard, The Austrian model of cooperation between the judges and the parties, en VAN RHEE, C.H. - Yulin, Fu (editores), Civil litigation in China and Europe: Essays on the role of the judge and the parties (Dordrecht, Springer, 2014), p. 179.

${ }^{85}$ Hess, Burkhard, JAuernig, Othmar, Manual de derecho procesal civil (trad. de Eduard Roig Molés, $30^{\text {a }}$ ed., Madrid, Marcial Pons, 2015), p. 38.

${ }^{86}$ VAn Rhee, C. H.; The development, cit. (n. 78), pp. 15-16.

${ }^{87}$ Ibíd., p. 13.

${ }^{88}$ Koller, C., Civil justice in Austrian-German tradition. The Franz Klein heritage and beyond, en UzELAC, Alan (ed.), Goals of civil justice and civil procedure in contemporary judicial systems (Springer International Publishing Switzerland, 2014), p. 36.

${ }^{89}$ Homburger, Adolf, cit. (n. 20), p. 24.

${ }^{90}$ Prüting, Hans, cit. (n. 68), p. 253.

${ }^{91}$ FrodL, Susanne, cit. (n. 25), p. 48. 
cuestión privada entre las partes. Su interferencia con los intereses de la colectividad tenía que manifestarse necesariamente en una nueva ordenación de las relaciones entre jueces y partes $^{92}$.

De este modo, la función de garantizar al individuo la manera más sencilla, libre y completa posible para la protección de sus derechos subjetivos, y el propósito adicional de tomar siempre en cuenta para la estructuración del enjuiciamiento sus repercusiones sobre la comunidad jurídica, si bien, aparentemente contradictorios, en verdad se prestan a una auténtica síntesis ${ }^{93}$.

\section{El rol activo del juez. El origen del moderno judicial case management}

En el sistema procesal civil austriaco de 1895, el juez civil dejó de estar "f u e r a del proceso, y sus poderes de dirección y de control fueron notablemente aumentados" 94 .

En efecto, la principal innovación del Código austríaco fue su énfasis en un papel más activo del juez ${ }^{95}$, para cumplir una doble función: por un lado, llegar a una decisión justa y correcta, al establecer la verdad ex officio y, por el otro, la gestión eficiente del caso como método eficaz para acelerar los procedimientos sin alterar su calidad. Así, garantizar la calidad de las decisiones y acelerar el procedimiento, no eran objetivos mutuamente excluyentes ${ }^{96}$.

La institución fundamental para este papel fue el contacto personal, o ral del juez con los litigantes ${ }^{97}$. En realidad, la teoría subyacente del modelo procesal austriaco era que un nuevo balance tenía que ser pensado entre los poderes de las partes y el juez ${ }^{98}$. Se trata de una coexistencia complementaria del principio dispositivo y el de autoridad delimitada del jue $z^{99}$.

El principio dispositivo es compañero de un sistema construido sobre derechos sustantivos que reconoce derechos privados. Esto garantiza al demandante el derecho a decidir si demanda o no, y cuando hacerlo; y para el demandado, el correspondiente derecho de admitir la demanda o resistirla. La destrucción del principio dispositivo causaría la caída del sistema entero de derechos y deberes privados. El principio de oficialidad, por otro lado, reconoce un interés público

\footnotetext{
${ }^{92}$ Fashing, Hans Walter; Liberalización y socialización del proceso civil, en Comunicación Nacional del Ponente Austriaco al Quinto Congreso Internacional de Derecho Procesal (1972), disponible en: http:// biblio.juridicas.unam. $\mathrm{mx} /$ revista/pdf/DerechoComparado/13/art/art2.pdf, p. 25 (consulta, 23 de octubre de 2015), p. 25.

${ }^{93}$ Ibíd., p. 24.

${ }^{94}$ Cappelletti, Mauro, El proceso civil, cit. (n. 71), p. 61.

${ }^{95}$ Chiovenda, Giuseppe, Ensayos de derecho procesal civil (trad. de Santiago Sentis Melendo, Buenos Aires, Ediciones Jurídicas Europa - América, Buenos Aires, 1949) II, pp. 142-143.

${ }^{96}$ Koller, Christian, Austrian national report (including additional information on Germany), en MALESHIN, Dmitry (ed.), Civil procedure in cross-cultural dialogue: Eurasia context: LAPL world conference on civil procedure, Conference Book (International Association of Procedural Law Russian Federation, 2012), pp. 146-147.

${ }^{97}$ CAPpelletti, Mauro, Social and political, cit. (n. 21), p. 854.

${ }^{98}$ Frodl, Susanne, cit. (n. 25), p. 45.

${ }^{99}$ Fairen Guillen, Víctor, Teoría general del derecho procesal (1 ${ }^{\text {a }}$ ed., México, D. F., Universidad Nacional Autónoma de México, 1992), p. 276 ss.
} 
predominante en la justa, rápida y económica determinación de las acciones civiles $^{100}$.

Al reestructurarse la posición del juez, su tarea ya no fue de simple vigilante de la legalidad del conflicto procesal, sino que de conductor del litigio ${ }^{101}$, y la piedra angular de este sistema procesal, la constituyó los poderes de gestión del juez ${ }^{102}$. Según esto, mientras las partes privadas son, generalmente, libres para disponer de sus derechos sustantivos en litigio, no lo son para controlar la manera como se desarrollará el procedimiento. El desarrollo de los procedimientos es un asunto de interés público, gobernado por los requisitos de una expedita y ordenada administración de justicia ${ }^{103}$.

Con todo, Cappelletti previene que el carácter social del código austríaco fue concebido por Klein de una manera demasiado vaga como para traducirlo en fórmulas jurídicas claras. Así, el problema de la articulación entre el principio dispositivo y los de sustanciación y dirección de oficio, no había encontrado todavía en la doctrina una solución totalmente satisfactoria ${ }^{104}$.

\section{Influencia del Código de Klein}

Las ideas de función social del proceso y el cambio del rol de los jueces fueron llevados a muchos códigos europeos. En Croacia, se adoptó prácticamente de un modo literal ${ }^{105}$. El Código Procesal Civil Húngaro de 1911, de Yugoslavia de 1929, de Checoslovaquia, la Ley de Dinamarca de 1916, de Noruega de 1915 y Suecia de 1942, son también hijos de la ordenanza austríaca ${ }^{106}$. Asimismo influyó en Polonia ${ }^{107}$ y Japón ${ }^{108}$. En Holanda, importantes propuestas de reforma recogieron las características del proceso civil austriaco, como el proyecto Gratama ${ }^{109}$. También en Alemania su influencia fue muy significativa, por ejemplo, en la Novelle

${ }^{100}$ Homburger, Adolf, cit. (n. 20), p. 24.

${ }^{101}$ FASHING, Hans Walter, cit. (n. 92), p. 27.

${ }^{102}$ Griss, Irmgard, cit. (n. 84), p. 179.

${ }^{103}$ Cappelletti, Mauro, Garth, Bryant G., cit. (n. 23), p. 23.

${ }^{104}$ Cappelletti, Mauro, El testimonio de la parte en el sistema de la oralidad. Contribución a la teoria de la utilización probatoria del saber de las partes en el proceso civil (La Plata, Editorial Platense, 2002) II, p. 84.

${ }^{105}$ Uzelac, Alan, Croatia: Omnipotent judges as the cause of procedural inefficiency and impotence, en VAn Rhee, C. H. - Yulin, Fu (eds.), Civil litigation in China and Europe: Essays on the role of the Judge and the Parties (Dordrecht, Springer, 2014), pp. 197-198.

${ }^{106}$ Goméz Lara, Cipriano, La oralidad en el proceso civil mexicano, en Revista del Instituto Colombiano de Derecho Procesal, 9/9 (1990), p. 180.

${ }^{107}$ Karolczyk, Bartosz, Pretrial as a part of judicial case management in Poland in comparative perspective, en Comparative Law Review, 15 (Nicolaus Copernicus University, 2013), pp. 184 ss.

${ }^{108}$ Matsumoto, Hiroyuki, The reception and transmission of the law of civil procedure in Japan. The experience in Japan, en Deguchi, Masahisa -STORME, Marcel (eds.), The reception and transmission of civil procedural law in the global society. Legislative and legal educational assistance to other countries in procedural law (International Association of Prodedural Law AIPL, Maklu Antwerpen - Apeldoorn, Maklu Publishers, 2008), p. XX.

${ }^{109}$ CADIET, Löic, The international sources, cit. (n. 50), pp. 266 ss. 
del Ministro de justicia Emminger de 1924, que convirtió al juez en responsable por la observancia de límites de tiempo ${ }^{110}$.

Además, las ideas de Klein sobre el rol activo del juez y la concepción del proceso civil, se recepcionaron en trabajos académicos que abrirían la senda de las reformas, como, por ejemplo, en Francia con el destacado procesalista Albert Tissier y sus discípulos René Morel y Henry Vizioz ${ }^{111}$. En este sentido, el profesor Cadiet indica que el Código de Procedimiento Civil de 1976, constituye un trabajo equilibrado entre la tradición liberal del Code de 1806 y la función social del proceso, heredado de las reformas procesales realizadas a finales del siglo XIX bajo la inspiración de Franz Klein (1854-1926) y predicada en Francia por Albert Tissier (1862-1925) en el umbral del siglo $\mathrm{XX}^{112}$. También influyó en la doctrina italiana, pero menos en el Codice di Procedura Civile de 1940, y a través de Italia, alguna influencia puede ser discernida sobre la península ibérica. Incluso el francés juge de la mise en etat, parece inspirado por el juez de la fase preparatoria austriaca ${ }^{113}$.

\section{Las Civil Procedure Rules de Inglaterra y Gales}

\section{Antecedentes. El surgimiento del proceso adversarial}

En Inglaterra el aumento de la autoridad del juez sobre el desarrollo de la litigación civil también vino de la mano del carácter oral del procedimiento. Sin embargo, mientras en Europa la oralidad fue una respuesta a las características del procedimiento del derecho común, en el mundo del common law fue un accidente de la historia ${ }^{114}$. Los jueces y abogados del siglo XVIII no sabían que el producto de su trabajo sería un nuevo sistema de adjudicación, el modelo adversarial ${ }^{115}$.

En efecto, en un principio el sistema medieval de jurados era de auto información ${ }^{116}$. Los reyes normandos emplearon jurados, que desarrollaban sus deberes basados en su conocimiento personal de los hechos ${ }^{117}$. En una época de pequeñas comunidades agrícolas intensamente interdependientes, los jurados fueron seleccionados del mismo vecindario con la esperanza que éste contendría personas que conocerían los hechos o que estarían bien posicionados para investigarlos por sus propios medios ${ }^{118}$. En consecuencia, el jurado medieval no fue a

${ }^{110}$ VAN RHEe, C. H., The development, cit. (n. 78), p. 17.

${ }^{111}$ CADIET, Löic, The international sources, cit. (n. 50), pp. 266 ss.

${ }^{112}$ CADIET, Löic, Introduction to French, cit. (n. 1), p. 366.

${ }^{113}$ En Francia el case management se identifica con la "mise en etat". Jeuland, Enmanuel, Case Management in France, en Van Rhee, C.H. - Yulin, Fu (eds.), Civil litigation in China and Europe: Essays on the role of the judge and the parties (Dordrecht, Springer, 2014), p. 350.

${ }^{114}$ Cappelletti, Mauro, Garth, Bryant G., cit. (n. 18), pp. 24 ss.

${ }^{115}$ Landsman, Stephan, The rise of the contentious spirit: Adversary procedure in eighteenth century England, en Cornell Law Review, 75/3 (1990), p. 520.

${ }^{116}$ Nokes, G. D., The English jury and the law of evidence, en Tulane Law Review, 31 (1956-1957), p. 154.

${ }^{117}$ Cappelletti, Mauro, Garth, Bryant G., cit. (n. 18), p. 25.

${ }^{118}$ Langbein, John H., The disappearance of civil trial in the United States, en Yale Law Journal, 122/3 (2012), p. 9 . 
la Corte a escuchar sino a hablar, no a oír evidencia, sino a entregar un veredicto formulado por anticipado ${ }^{119}$.

No obstante, a lo largo de la Edad Media, el jurado dejó de funcionar de esta manera por razones complejas, incluidas las dislocaciones demográficas cataclísmicas tras la peste negra, y los efectos de la urbanización en la producción de relaciones sociales más impersonales ${ }^{120}$. En el tiempo, el uso de información extrajudicial sobre los hechos del juicio fue perdiendo importancia, la interrogación de los testigos por las partes se convirtió en la forma por la cual el jurado era informado ${ }^{121}$, quienes debían basar su veredicto sólo en la prueba producida en la Corte $^{122}$.

Este jurado pasivo requirió un procedimiento de instrucción del tribunal, por medio del cual, los testigos extraños le entregarían información, lo que dio origen al derecho probatorio (evidence law). El juez, en consecuencia, desarrolló un poder para advertir el mérito de la prueba, lo que fue bien recibido por el jurado ${ }^{123}$. Al mismo tiempo, se mantuvo el carácter lego de los jurados, el procedimiento oral y necesariamente concentrado ${ }^{124}$.

El juicio por jurados había sido transformado en un procedimiento adversarial $^{125}$, caracterizado por el control de las partes en la investigación y presentación de pruebas, y por un juez pasivo que escuchaba a las partes, tomando su decisión basado en lo que había oído ${ }^{126}$.

En Inglaterra, bajo la concepción adversarial, los jueces no debían participar en la preparación de los casos para el juicio. La independencia judicial los obligaba a permanecer desvinculados y desapasionados de la disputa, para asegurar la justicia e imparcialidad de los resultados. El curso tradicional del litigio, por lo tanto, descansó en manos de los litigantes ${ }^{127}$.

2. La reforma de Lord Woolfy el debilitamiento del adversary system

Inglaterra tiene una gran tradición en materias de reformas a la justicia. Más de

${ }^{119}$ LANGBEIn, John H., Historical foundations of the law of evidence: A view from the Ryder sources, en Columbia Law Review, 96/5 (1996), p. 1170.

${ }^{120}$ LangBein, John, The demise of trial in american civil procedure: How it happened, is it convergence with european civil procedure?, en VAN RHEE, C. H. - UzELAC, A. (eds.), Truth and efficiency in civil litigation fundamental aspects of fact-finding and evidence-taking in a comparative context (Intersentia Cambridge - Antwerp - Portland, 2012), p. 123.

${ }^{121}$ Landsman, Stephan, From Gilbert to Bentham: The reconceptualization of evidence theory, en Wayne Law Review, 36 (1990), p. 1172.

${ }^{122}$ Morgan, Edmund M., The jury and the exclusionary rules of evidence, en The University of Chicago Law Review, 4/2 (1937), pp. 247 ss.

${ }^{123}$ LANGBEIN, John, Bifurcation and the bench: The influence of the jury on english conceptions of the judiciary, en Brand, Paul - Getzler, Joshua (eds.), Judge and judging in the history of common law and civil law: From antiquity to modern times (Cambridge University Press, 2012), p. 77.

${ }^{124} \mathrm{La}$ oralidad fue reforzada por la división de la profesión legal con barrister especializados en litigios orales. Véase Cappelletti, Mauro, Garth, Bryant G., cit. (n. 23), p. 25.

${ }^{125}$ Morgan, Edmund M., cit. (n. 121), pp. 247 ss.

${ }^{126}$ SwARD, Ellen E., Values, ideology and the evolution of the adversary system, en Indiana Law Journal, 64/2 (1989), p. 302.

${ }^{127}$ Vorrasi, Kenneth M., England's reform to alleviate the problems of civil process: A comparison of judicial case management in England and the United States, en Journal of Legislation, 30/2 (2004), p. 362. 
60 reportes o comisiones desde 1851 han propuesto reformas al procedimiento inglés, tanto en materias civiles como criminales, con un mismo objetivo: reducir la complejidad de la litigación, los costos y demoras ${ }^{128}$. Sin embargo, la mayor transformación se inició sólo con los Informes de Lord Woolf ${ }^{129}$.

En efecto, en marzo de 1994, Lord Chancellor (Lord Mackay of Clashfern) designó a Lord Harry Woolf, para que dirigiera un estudio que buscara la manera de mejorar la accesibilidad y velocidad del sistema, y de reducir sus $\operatorname{costos}^{130}$. Para estos fines, Lord Woolf realizó una extensa investigación tanto en Inglaterra como en otras jurisdicciones ${ }^{131}$, entregando sus propuestas en dos reportes denominados Access to Justice (Acceso a la Justicia) ${ }^{132}$, Interim Report de 1995 y Final Report de $1996^{133}$, proveyendo de las bases para profundos cambios en la litigación civil ${ }^{134}$.

Lord Woolf calificó la justicia civil como muy cara, lenta y compleja ${ }^{135}$. Además, la cultura adversarial, entendida como ausencia de control por los jueces, fue identificada como la raíz de muchos problemas, donde la litigación era vista como un campo de batalla sin reglas ${ }^{136}$. En este sentido, el reporte de Lord Woolf, fomentaba a las partes a adoptar una nueva actitud, en la cual, la cooperación fue enfatizada ${ }^{137}$.

Los informes Access to Justice se basan en ocho principios que el sistema de justicia civil debe cumplir: (a) ser justo en los resultados que ofrece; (b) ser justos en el trato hacia los litigantes; (c) ofrecer procedimientos adecuados a un costo razonable; (d) tratar los casos con velocidad razonable; (e) ser comprensible para aquellos que lo utilizan; (f) responder a las necesidades de los que lo utilizan; (g)

${ }^{128}$ En 1908 Gorell Committee; 1913 St. Alawyn Committee; 1922 Swift Committee; en 1932, 2 intentos de reforma: Handworth Committee y Peel Commision; 1947 Evershed Committee; 1968 Winn Committee. Cantley hizo un reporte en 1979, Oliver hizo uno para la division de la Chancery que se publicó en 1981. En 1988 The Civil Justice Review. En 1994 Heilbron/Hodge, y en 1997 Middleton. Clarke, Anthony, The Woolf reforms: A singular event or an ongoing process?, en DWYER, Déirdre (ed.), The civil procedure rules ten years on (University of Oxford, 2009), pp. 34 ss.

${ }^{129}$ Van Rhee, C. H., The development, cit. (n. 78), p. 22.

${ }^{130}$ Stapper, Gary - Kelly, David, The English legal system (Twelfth ed., Routledge, 2011), p. 332 .

${ }^{131}$ ElLiotT, Catherine - Quinn, Frances, English legal system (Tenth ed., Pearson Education Limited, 2009), p. 516.

${ }^{132}$ Finch, Emily -FAfinsky, Stefan, English legal system (Forth ed., Pearson Education Limited, 2013), p. 136.

${ }^{133}$ Una primera reacción académica al Interim Report puede verse en: ZuCKERMAN, A. A. S. - Cranston, Ross (editores), Reform of civil procedure: Essays on "acess to justice" (Oxford University Press, 1996).

${ }^{134}$ Dwyer, Déirdre, Introduction, en Dwyer, Déirdre (ed.), The civil procedure rules ten years on (Oxford, Oxford University Press, 2009), pp. 2-3.

${ }^{135}$ Woolf, Harry, Final report, section I, overview, disponible en http://webarchive. nationalarchives.gov.uk/+/http:/www.dca.gov.uk/civil/final/contents.htm (consulta, 04 de febrero de 2018).

${ }^{136}$ WoOLF, Harry, Access to Justice, Interim Report. The problems and their causes, disponible en http://webarchive.nationalarchives.gov.uk/+/http://www.dca.gov.uk/civil/interim/chap3. htm (consulta, 4 de febrero de 2018).

${ }^{137}$ VAn Rhee, C. H., The development, cit. (n. 78), p. 22. 
proporcionar la mayor certeza que la naturaleza de los casos particulares permite; y (h) ser eficaz: los recursos adecuados y organizados ${ }^{138}$.

Estos principios proveyeron el marco de las Civil Procedure Rules que gobiernan la litigación, y que entraron en vigor el 26 de abril de $1999^{139}$. En estas se proponen los siguientes objetivos: (1) acelerar la justicia civil, (2) hacer más accesible el proceso civil a la gente común, (3) simplificar el lenguaje, (4) promover los acuerdos (5) hacer que los litigios sean más eficientes y menos costosos evitando el recurso excesivo y desproporcionado a los procedimientos ${ }^{140}$.

Con todo, Lord Woolf afirmó que el objetivo de su reforma no fue socavar el carácter adversarial del sistema de justicia civil inglés ${ }^{141}$, por lo que se mantuvo la responsabilidad de las partes y los profesionales del derecho para manejar sus casos, pero ahora en un entorno gestionado por los tribunales y por normas que centran los esfuerzos en las cuestiones clave del caso, en lugar de permitir que todos los temas sean considerados, sin tener en cuenta los gastos y el tiempo ${ }^{142}$.

En efecto, la reforma, si bien eliminó la autonomía de las partes sobre la progresión del caso, dejó la estructura fundamental del sistema adversarial, sin tocar ${ }^{143}$. Los aspectos que se conservaron son: (1) La decisión de iniciar el proceso; (2) la selección de un remedio o reparación; (3) La decisión unilateral de una partes de retirar una demanda o una defensa; o de resolver el caso por acuerdo; (4) la decisión de ejecutar una sentencia u otra orden; (5) La decisión de deducir una apelación ${ }^{144}$.

3. La relación instrumental entre el case management y el overriding objective (objetivo predominante). Un concepto de proceso

Las CPR giran en torno del case management, lo que no sólo supone una intervención más activa del juez en un proceso particular, sino también el equilibrio de los recursos entre el tratamiento de éste y la sustentabilidad de todo el sistema judicial.

Este case management ya era conocido en la propia Inglaterra, y en la experiencia comparada ${ }^{145}$. En Inglaterra, aparece como una respuesta a los excesos del

\footnotetext{
${ }^{138}$ Woolf, Harry, Access to Justice: Final report, section I, overview, cit. (n. 135) (consulta, 4 de febrero de 2018).

${ }^{139}$ Andrews, Neil, English civil justice in a competitive world, en DeGuchi, Masahisa - STORME, Marcel (eds.), The reception and transmission of civil procedural law in the global society. Legislative and legal educational assistance to other countries in procedural law (International Association of Prodedural Law AIPL, Maklu Antwerpen - Apeldoorn, Maklu Publishers, 2008), p. 290.

${ }^{140}$ Andrews, Neil, English civil procedure: A synopsis, en Ritsumeikan Law Review, 25 (2008), p. 31.

${ }^{141}$ Access to Justice - Final Report, Section II, Case Management, Chapter I, Introduction, p. 1, disponible en http://webarchive.nationalarchives.gov.uk/20040722070027/http://www.dca.gov.uk/civil/ final/sec2a.htm\#cl (consulta, 3 de febrero de 2018).

${ }^{142}$ Woolf, Harry, Civil justice in the United Kingdom, en The American Journal of Comparative Law, 45/4 (1997), p. 726.

${ }^{143}$ Sorabj, John, Austerity's effect on English civil justice, en Erasmus Law Review, 8/4 (2015), p. 165.

${ }^{144}$ Andrews, Neil, The new English procedure rules, en VAN RHEE, C. H. (ed.), Europeans traditions in civil procedure (Intersentia Antwerpen - Oxford, 2005), p. 167.

${ }^{145}$ Fue propuesto por primera vez en 1826 por la First Chancery Reform Commission. Clakke, Anthony, cit. (n. 128) (University of Oxford, 2009), p. 44. En Inglaterra, era conocido en la
} 
sistema adversarial tradicional ${ }^{146}$, que generaba un aumento innecesario de costos y el retardo en la resolución del proceso ${ }^{147}$. Por esta razón, Woolf señaló que la base para la reforma sería "un sistema en el que los tribunales, con asistencia de los litigantes, se encargaría del case management (gestión de los casos), incluyendo determinar qué procedimientos eran adecuados para cada caso, el establecimiento de calendarios realistas, y la garantía de que los procedimientos y horarios se cumplirían" ${ }^{148}$.

Como se observa, Lord Woolf concluyó que la responsabilidad final por el control de la litigación debía pasar de los litigantes y sus asesores legales a las Cortes $^{149}$, con el propósito que los tribunales traten los casos de manera justa y a un costo proporcional, por lo que puede describirse como una reforma instrumental $^{150}$. Según esto, el case management, no es un fin en sí mismo.

En efecto, el case management requiere un propósito, y éste se encuentra definido por el overriding objective. Así, la regla 1.1 de las CPR dispone que: "1) Las presentes Reglas constituyen un nuevo código de procedimiento con el objetivo predominante de permitir que el tribunal se ocupe de los asuntos de manera justa y a un costo proporcional. (2) La tramitación justa y a un costo proporcional de un caso incluye, en la medida de lo posible, (a) Asegurar que las partes están en un pie de igualdad; (b) Ahorrar costos; (c) Tratar los casos de maneras que sean proporcionadas (i) $A$ la cantidad de dinero envuelta (ii) $A$ la importancia del caso (iii) $A$ la complejidad de los asuntos; y (iv) A la posición financiera de cada parte (d) Asegurando que sean conducido con justicia y con toda prontitud (e) Asignando a ellos una parte apropiada de los recursos de la corte, teniendo en cuenta la necesidad de asignar recursos para otros casos. (f) Imponer el cumplimiento de las normas, instrucciones prácticas y órdenes"151.

$\mathrm{El}$ profesor Sorabji observa que el case management sólo genera un cambio, desde el control de las partes sobre el ritmo del litigio hacia el control judicial. En otras palabras, transfiere el volante a un nuevo conductor, sin embargo, por si sólo, nada dice sobre el destino hacia el cual lo conduce ${ }^{152}$. En realidad, son los objetivos del sistema de justicia los que guían y definen cómo y para qué el case management debe ser entendido y utilizado. En este sentido, el overriding objective es verdaderamente innovador, porque proporciona un nuevo controlador del case management, con un nuevo destino, confiriendo a la litigación un nuevo propósito, radicalmente diferente del anterior a las reformas de Woolf. Un nuevo concepto de justicia se introdujo en el procedimiento civil inglés, comprometido con la

Commercial Court, en el Official Referees' Court, y en las cortes de condado. También en países como Escocia, Estados Unidos, Canadá, Australia y Nueva Zelanda. Cfr. Access to Justice - Final Report, Section II, cit. (n. 141), p. 2, (consulta, 3 de febrero de 2018). También Woolf estudió el cade management francés. Jeuland, Enmanuel, Case Management in France, cit. (n. 113), p. 350,

${ }^{146}$ LEGG, Michael, Case management and complex litigation (Federation Press, 2011), pp. 2-3.

${ }^{147}$ Ibíd.

${ }^{148}$ WoOLF, Harry, Final report, section I, overview, cit. (n. 135).

149 Access to Justice - Final Report, Section II, cit. (n. 141), p. 1, (consulta 3 de febrero de 2018).

${ }^{150}$ Neuberger, David, Framing a new procedural culture, en Civil Justice Quaterly, 34/3 (2015), p. 238.

${ }^{151}$ Cfr. Civil Procedure Rule, Part 1.1.

${ }^{152}$ SORABJI, John, The road to new street station: Fact, fiction and the overriding objective, en European Business Law Review, 23/1 (2012), p. 78. 
proporcionalidad en lugar de, como fue previamente el caso, un compromiso puro con el logro de lo que Woolf describió como justicia sustantiva, es decir, justicia sobre el fondo ${ }^{153}$.

Según Zuckerman, este overriding objective constituye un concepto de proceso, ya que proporciona a los tribunales un conjunto de políticas que deben guiar el case management ${ }^{54}$. Estos principios se basan en la noción que la administración de justicia es un ejercicio práctico, no meramente académico, que debe necesariamente tener en cuenta las inevitables limitaciones prácticas de los recursos y del tiempo. Por consiguiente, la solución de los litigios civiles debe ser gestionada de manera proporcional a las necesidades del caso y a los recursos de que disponen los tribunales ${ }^{155}$.

Este overriding objective (1) no contiene un compromiso singular de logro sustantivo de la justicia; por el contrario, contiene un compromiso igual tanto con la justicia sustantiva como con la procesal; (2) no trata la economía y la eficiencia como medios para asegurar una justicia sustantiva a través del proceso legal; en cambio, considera la economía y la eficiencia como principios que ponen un límite al compromiso de asegurar una justicia sustantiva; y (3) requiere un enfoque riguroso por parte de los tribunales con los litigantes que no cumplen con las obligaciones procesales, y que, por lo tanto, no hacen un uso adecuado de un proceso justo y proporcional ${ }^{156}$.

De este modo, el overriding objective, introdujo en el procedimiento civil inglés, la idea motriz que la justicia implica no sólo entregar sentencias que sean correctas en los hechos y el derecho, sino también que debe hacerlo en un plazo razonable, mediante el uso proporcional de los recursos judiciales y de los litigantes ${ }^{157}$. Esta proporcionalidad se expresa considerando el valor, importancia y complejidad del caso, tanto en la organización de los diferentes niveles de procedimiento -small claim, fast track y multy track-, como en el ejercicio de los poderes de case management de los tribunales ${ }^{158}$.

La incorporación en el overriding objective que el tribunal se ocupe de los asuntos "a un costo proporcional", fue establecido tras el Informe de Lord Jackson, para enfatizar la preocupación por la proporcionalidad de los costos, y un enfoque

${ }^{153}$ Ibíd.

${ }^{154}$ Zuckerman, Adrian, Court adjudication of civil disputes: A public service that needs to be delivered with proportionate resources, within a reasonable time and at reasonable cost, disponible en http://www. aija.org.au/ac06/Zuckerman.pdf, p. 8, (consulta, 18 de febrero de 2018), p. 8.

${ }^{155}$ Zuckerman, Adrian, Conference on "The ALI-UNIDROIT principles and rules of transnational civil procedure", hosted by the British Institute of International and Comparative Law, London May 24, 2002, en Civil Justice Quarterly, (2002), p. 323.

${ }^{156}$ SORABJI, John, The road to new street station: Fact, fiction and the overriding objective, en European Business Law Review, 23/1 (2012), pp. 88-89.

${ }^{157}$ ZuCKERMAn, A.A.S., The revised CPR 3.9: a coded message demanding articulation, en ZuCKERMAN, A.A.S. (ed.), Civil Justice Quarterly, Special Issue: The implementation of Sir Rupert Jackson's review of civil litigation costs, 32/2 (2013), p. 128.

${ }^{158}$ Andrews, Neil, The three paths of justice: Court proceedings, arbitration and mediation in England (Springer, 2012), p. 5. 
más robusto para el desarrollo del procedimiento bajo la conducción del juez ${ }^{159}$. La proporcionalidad, según Jackson, es un concepto de textura abierta, y, cuya esencia, en el proceso civil del Reino Unido es "[...] que los fines no necesariamente justifican los medios. La ley facilita la consecución de objetivos legítimos, pero sólo en la medida en que esos objetivos justifiquen las cargas que se imponen a los demás" $" 160$.

En relación a éste último aspecto, es muy relevante que la responsabilidad del juez en el control del caso individual, considere el conjunto de los $\operatorname{casos}^{161}$. En efecto, tradicionalmente, en Inglaterra el rol del tribunal siempre se limitó a tratar con justicia cada caso individual, sin que tuviera ninguna obligación de garantizar positivamente que la totalidad del proceso de litigación se gestionare de una manera justa ${ }^{162}$. Con las CPR, se introdujo una preocupación por el conjunto de casos, disponiendo la regla 1.1 (2) (e) que "ocuparse de un caso justamente y a un costo proporcional incluye, en la medida de lo posible, asignarle una parte apropiada de los recursos del tribunal, teniendo en cuenta la necesidad de asignar recursos a otros casos" ${ }^{163}$. Lord Woolf, en una sentencia redactada por el mismo, después de la vigencia de las CPR, consignó sobre el punto que: "La responsabilidad de un juez hoy en el manejo adecuado de la litigación requiere que, al ejercer su discreción de acuerdo con el objetivo predominante contenido en la Parte 1 de las CPR, considere el efecto de su decisión sobre la litigación en general"164. De esto se sigue que la justicia es más que la justicia en los casos individuales, es la justicia en todos los $\operatorname{casos}^{165}$.

El cambio revolucionario de filosofía, se refiere a una nueva teoría de la justicia denominada como justicia proporcional, en la cual la justicia sustantiva no es el único objetivo. Poner la justicia sustantiva por encima de todo, representa una elección particular, ofrecer justicia sin reparar en gastos para los pocos que puedan permitírselo. Esto genera que la justicia sea denegada, en todo o en parte, a grandes sectores de la población ${ }^{166}$.

En suma, los dos aspectos más significativos de las reformas de Woolf son la introducción del overriding objective, con su compromiso con la proporcionalidad y un activo case management de los casos por los jueces. El primero prescribe cómo la litigación debe ser conducida por los tribunales y los litigantes. El segundo,

\footnotetext{
${ }^{159}$ O’Hare, John - Browne, Kevin, Civil litigation (17th ed., London, Sweet \& Maxwell, 2015), p. 4.

${ }^{160}$ JaCKSOn, Rupert, Review of civil litigation costs. Final report (The Stationery Office, United Kingdom, 2009), p. 36.

${ }^{161}$ Grevling, Katharine, CPR $r$ 32.1(2): Case management tool or broad exclusionary power?, en DWYER, Déirdre (ed.), The civil procedure rules ten years on (University of Oxford, 2009), pp. 261 ss.

${ }^{162}$ JoHnson, Adam, Civil litigation in England - the impact of Lord Woolf's reforms, en Journal of International Banking Law (1999), p. 201.

${ }^{163}$ CPR, PART 1 Rule 1.1 (2) (e) The overriding objective 1.1, disponible en World Wide Web: https://www.justice.gov.uk/courts/procedure-rules/civil/rules/part01.

${ }^{164}$ Zuckerman, Adrian, Editor's Note. Must a fraudulent litigant be allowed to think: if the fraud is successful, I will gain much; if it is not, I will still recover my legitimate claim?, en Civil Justice Quaterly, $30 / 1$ (2011), p. 8.

${ }^{165}$ Clarke, Anthony, cit. (n. 128), p. 45.

${ }^{166}$ ZuCKerman, Adrian, Justice in crisis cit. (n. 11), p. 17.
} 
proporciona los medios para que los tribunales lleven a efecto ese objetivo, con la ayuda de las partes $^{167}$.

\section{Influencia de las Civil Procedure Rules, en la perspectiva del case management}

Las CPR se han utilizado como un ejemplo para la reforma procesal civil ${ }^{168}$. El Código de Procedimiento Civil Portugués, contiene un principio de economía procesal similar al adoptado por las CPR. En Francia, donde la teoría tradicional era que el proceso civil pertenece a las partes, el judicial tiene ahora extensos poderes para controlar la litigación civil. Zuckerman considera que las ideas de flexibilidad y proporcionalidad no son muy diversas a los de las reglas inglesas. Una observación similar podría ser hecha acerca de las tendencias en España y Japón ${ }^{169}$

En el mismo sentido, la proporcionalidad y el case management han tenido una importante recepctividad en la doctrina europea continental ${ }^{170}$. Así, por ejemplo, el profesor Remo Caponi refiriéndose específicamente al modelo inglés, ha propuesto profundizar sobre la proporcionalidad en el proceso civil, la que vincula con la tendencia europea de establecer un proceso de cognición completo con cierta elasticidad, que conlleva el desarrollo más deseable para el caso concreto, a elección del juez, entre los modelos de tratamiento múltiple, en relación con el tipo de disputa concreta sometido a su examen ${ }^{171}$. El profesor Taruffo, entregando como ejemplo, los tracks de las CPR inglesas, considera que adaptar el instrumento procesal a las necesidades de cada caso, según la complejidad de la controversia, una alternativa eficiente para evitar el despilfarro de recursos, agregando que las formas del procedimiento deben configurarse e interpretarse de manera elástica y flexible, por lo que "...el dominus de esta adaptación del proceso a las exigencias de cada caso, no podría ser sino el juez, quien debería estar dotado de los poderes necesarios para un efectivo case management ${ }^{\prime 172}$.

\section{CONCLUSIONES}

En Europa, la tendencia evolutiva de entregar un mayor control de la litigación

${ }^{167}$ Neuberger, David, Docketing: Completing case management's unfinished revolution, en Ninth Lecture in Implementation Programme Solicitors' Costs Conference (London, 2012), p. 5, disponible en World Wide Web: https://www.judiciary.gov.uk/wp-content/uploads/JCO/Documents/ Speeches/mor-speech-solicitors-cost-conference-lecture-feb2012.pdf (consulta, 13 de febrero de 2018).

${ }^{168}$ Dwyer, Déirdre, Introduction, cit. (n. 134), p. 5.

${ }^{169}$ Zuckerman, Adrian A.S., Justice in crisis, cit. (n. 11), p. 48.

${ }^{170} \mathrm{Al}$ menos tres Congresos Mundiales de Derecho Procesal se han referido al case management. Véase cita 19.

${ }^{171}$ CAPONI, Remo, II principio di proporzionalitá nella giustizia civile: prime note sistematiche, en Rivista trimestrale di diritto e procedura civile (2010), p. 6, disponible en World Wide Web: https:// www.academia.edu/211692/R._Caponi._Il_principio_di_proporzionalit\%C3\%A0_nella_ giustizia_civile_prime_note_sistematiche_2010 (consulta, 3 de mayo de 2017). También en Italia véase FiCARELLi, Beatrice, Fase preparatoria del proceso civile e case management giudiziale, (Edizione Scientifiche Italiane, Napoli, 2011).

${ }^{172}$ TARuffo, Michele, Una alternativa a las alternativas: Modelos de resolución de conflictos, en Páginas sobre justicia civil (Madrid, Marcial Pons, 2009), p. 121. 
civil a los jueces en cooperación con las partes, ha desembocado en el judicial case management, el que constituye, en la actualidad, su configuración conceptual más moderna.

Esta perspectiva nos revela que la mayor entrega de confianza y responsabilidades a los jueces, se asocia a una ampliación de los objetivos del proceso, como la función social del proceso civil austriaco o el overriding objective del Reino Unido.

De este modo, el case management se configura como un instrumento de eficiencia que permite que los diversos objetivos del sistema de justicia, puedan concretarse en los procedimientos. Se trata, en definitiva, de una configuración del rol de los jueces, que los involucra en el manejo eficiente del conjunto de la litigación, lo que implica una superación y alejamiento del tradicional rol del juez, determinado por el carácter individualista y el contenido privado del proceso civil.

\section{Bibliografía Citada}

Allemeersch, B., The belgian perspective on case management in civil litigation, en VAN RHEE, C. H. (ed.), Judicial case management and efficiency in civil litigation (Intersentia Antwerp - Oxford - Portland, 2008), pp. 79-92.

Andrews, Neil, A new civil procedural code for England: Party control "going, going, gone", en Civil Justice Quarterly, 19 (2000), pp. 19-38.

- Andrews On Civil Processes (Intersentia Cambridge, Antwerp Portland, 2013), I.

—English civil justice in a competitive world, en Deguchi, Masahisa - Storme, Marcel (eds.), The reception and transmission of civil procedural law in the global society. Legislative and legal educational assistance to other countries in procedural law (AIPL, Maklu Antwerpen - Apeldoorn, Maklu Publishers, 2008), pp. 290-309.

- English civil procedure. Fundamentals of the new civil justice system (New York, Oxford University Press, 2003).

- English civil procedure: A synopsis, en Ritsumeikan Law Review, 25 (2008), pp. 25-61.

- The new English procedure rules, en Van Rhee, C. H. (ed.), Europeans traditions in civil procedure (Intersentia Antwerpen - Oxford, 2005), pp. 161-181.

- The three paths of justice: Courtproceedings, arbitration and mediation in England (Springer, 2012)

BAKER, Wallace R., French judgments subject to immediate appeal, en Law and Contemporary Problems, 47/3 (1984), pp. 17-34.

Berizonce, Roberto Omar, Recientes tendencias en la posición del juez. Relación general, en BerIzOnCE, Roberto Omar (coord.), El juezy la magistratura (Tendencias en los albores del siglo XXI) (Santa Fe, Rubinzal Culzoni Editores, 1999), pp. 15-58.

CADIET, Löic, Case management judiciaire et déformalisation de la procédure, en Revue Française d'Administration Publique 125/1 (2008), pp. 133-150.

— Introduction to french civil justice system and civil procedural law, en Ritsumeikan Law Review, 28 (2011), pp. 331-393.

— La justicia civil francesa entre eficiencia y garantías, en Civil Procedure Review, 3/4 (2013), pp. 25-50.

- Sources and destiny of french civil procedure in a globalized world, en Picker, Coli B. SeIDman, Guy I. (eds.), The dynamism of civil procedure. Global trends and developments, en Ius Gentium: Comparative Perspective on Law and Justice, 48 (Springer International Publishing, 2016), pp. 63-84. 
— The international sources of french civil procedure, en Deguchi, Masahisa -Storme, Marcel (eds.), The reception and transmission of civil procedural law in the global society. Legislative and legal educational assistance to other countries in procedural law (AIPL, Maklu Antwerpen - Apeldoorn, Maklu Publishers, 2008), pp. 261-274.

Cappelletti, Mauro, Garth, Bryant G., International encyclopedia of comparative law XVI (Dordrecht, Boston, Lancaster, J.C.B Mohr (Paul Siebeck), Tubingen y Martinus Nijhoff Publishers, 1987), I.

CAPpelletti, Mauro, El proceso civil en el derecho comparado. Las grandes tendencias evolutivas (trad. de Santiago Sentis Melendo, $1^{\text {a }}$ ed., Buenos Aires, Ediciones Jurídicas Europa- América S.A., 1973).

- El testimonio de la parte en el sistema de la oralidad. Contribución a la teoría de la utilización probatoria del saber de las partes en el proceso civil (La Plata, Editorial Platense, 2002) II.

- La oralidad y las pruebas en el proceso civil (trad. de Santiago Santis Melendo, Buenos Aires, Ediciones Jurídicas Europa - América S.A., 1972).

- Social and political aspects of civilprocedure: Reforms and trends in western and eastern Europe, en Michigan Law Review, 69/5 (1971), pp. 847-850.

- Vindicating the public interest through the courts: A comparativist's contribution, en Buffalo Law Review, 25 (1975-1976), pp. 643- 690.

- Who watches the watchmen? A comparative study on judicial responsibility, en American Journal of Comparative Law, 31/1 (1983), pp. 1-62.

CARPI, Federico, Le riforme del processo civile in Italia verso il XXI secolo, en Rivista Trimestrale di Diritto e Procedura Civile (Milano, Giuffré Editore, 2000), pp. 105-126.

CEPEJ, Report on "European judicial systems - Edition 2014 (2012 data): efficiency and quality of justice", disponible en World Wide Web: http://www.coe.int/t/dghl/ cooperation/cepej/evaluation/2014/Rapport_2014_en.pdf. (consulta, 23 de marzo de 2017).

Chiovenda, Giuseppe, Ensayos de derecho procesal civil (trad. de Santiago Sentis Melendo, Buenos Aires, Ediciones Jurídicas Europa - América, Buenos Aires, 1949) II.

Civil Procedure Rules disponibles en World Wide Web: http://www.justice.gov.uk/ courts/procedure-rules/civil (consulta, 22 de marzo de 2018).

Clarke, Anthony, The Woolf reforms: A singular event or an ongoing process?, en DwYER, Déirdre (ed.), The civil procedure rules ten years on (University of Oxford, 2009), pp. 33-50.

Damaska, Mirjan, The common law/ civil law divide: Residual truth of a misleading distinction, en Chase, Oscar G. - Walker, Janet (eds.), Common Law, Civil Law and the Future of Categories (Markham, LexisNexis, 2010), pp. 3-22.

DWYER, Déirdre, Introduction, en DWYER, Déirdre (ed.), The civil procedure rules ten years on (Oxford, Oxford University Press, 2009), pp. 1-36.

Eliıtot, Catherine, Quinn, Frances, English legal system (Tenth ed., Pearson Education Limited, 2009).

Engelmann, Arthur et al, History of continental civil (trad. de Robert Wyness Millar, Boston, Little Brown \& Co., 1927).

Fairen Guillen, Víctor, Teoría general del derecho procesal (1ª ed., México, D. F., Universidad Nacional Autónoma de México, 1992).

FAsHING, Hans Walter, Liberalización y socialización del proceso civil, en Comunicación Nacional del Ponente Austriaco al Quinto Congreso Internacional de Derecho procesal (1972), disponible en World Wide Web: http://biblio.juridicas.unam.mx/revista/pdf/ DerechoComparado/13/art/art2.pdf, (consulta, 23 de octubre de 2017). 
FiCARELl, Beatrice, Fase preparatoria del proceso civile e case management giudiriale (Edizione Scientifiche Italiane, Napoli, 2011).

Finch, Emily, Fafinsky, Stefan, English legal system (Forth ed., Pearson Education Limited, 2013).

Frodl, Susanne, The heritage of the Franz Klein reform of Austrian civil procedure in 1895 1896, en Civil Justice Quarterly, 31/1 (2012), pp. 43-67.

Garth, Bryan, Franz Klein, Mauro Cappelletti, and the mission of comparative procedural scholars: opening lecture for Intemational Association of Procedural Law, en Revista de Derecho de la Pontificia Universidad Católica del Perú, 52 (1999), pp. 555-563.

Goméz Lara, Cipriano, La oralidad en el proceso civil mexicano, en Revista del Instituto Colombiano de Derecho Procesal, 9/9 (1990), pp. 1-17.

Grevling, Katharine, CPR r 32.1(2): Case management tool or broad exclusionary power?, en DWYER, Déirdre (ed.), The civil procedure rules ten years on (University of Oxford, 2009), pp. 249-268.

GRISs, Irmgard, The Austrian model of cooperation between the judges and the parties, en Van Rhee, C.H. - Yulin, Fu (eds.), Civil litigation in China and Europe: Essays on the role of the judge and the parties (Dordrecht, Springer, 2014), pp. 179-184.

Hess, Burkhard, Jauernig, Othmar, Manual de derecho procesal civil (trad. de Eduard Roig Molés, $30^{\mathrm{a}}$ ed., Madrid, Marcial Pons, 2015).

Homburger, Adolf, Functions of orality in Austrian and American civil procedure, en Buffalo Law Review, 20 (1970), pp. 9-39.

JACKSON, Rupert, Review of civil litigation costs. Final report (The Stationery Office, United Kingdom, 2009).

Jolowicz, J. A., Civil litigation: What's it for?, en Cambridge Law Journal, 67/3 (2008), pp. 508-520.

- On civil procedure (First ed., Cambridge University Press, 2000).

KarolczyK, Bartosz, Pretrial as a part of judicial case management in Poland in comparative perspective, en Comparative Law Review, 15 (Nicolaus Copernicus University, 2013), pp. 151-188.

Koller, C., Civil justice in Austrian-German tradition. The Franz Klein heritage and beyond, en UzELAC, Alan (ed.), Goals of civil justice and civil procedure in contemporary judicial systems (Springer International Publishing Switzerland, 2014), pp 35-59.

- Austrian national report (including additional information on Germany), en MALESHIN, Dmitry (ed.), Civil procedure in cross-cultural dialogue: Eurasia context: LAPL world conference on civil procedure, Conference Book (International Association of Procedural Law Russian Federation, 2012), pp. 136-156.

Landsman, Stephan, From Gilbert to Bentham: The reconceptualization of evidence theory, en Wayne Law Review, 36 (1990), pp. 1149-1186.

- The rise of the contentious spirit: Adversary procedure in eighteenth century England, en Cornell Law Review, 75/3 (1990), pp. 497-609.

LANGBEIN, John H., Historical foundations of the law of evidence: A view from the Ryder sources, en Columbia Law Review, 96/5 (1996), pp. 1168-1202.

- The disappearance of civil trial in the United States, en Yale Law Journal, 122/3 (2012), pp. 1-51.

- Bifurcation and the bench: The influence of the jury on english conceptions of the judiciary, en Brand, Paul - GetzLER, Joshua (eds.), Judge and judging in the history of common law and civil law: From antiquity to modern times (Cambridge University Press, 2012), pp. 67-82.

- The demise of trial in american civil procedure: How it happened, is it convergence with european 
civil procedure?, en Van Rhee, C. H. - Uzelac, A. (eds.), Truth and efficiency in civil litigation fundamental aspects of fact-finding and evidence-taking in a comparative context (Intersentia Cambridge - Antwerp - Portland, 2012), pp. 117-164.

LegG, Michael, Case management and complex litigation (Federation Press, 2011).

LenHoff, Arthur, The law of evidence: A comparative study based essentially on Austrian and New York law, en The American Journal of Comparative Law, $3 / 3$ (1954), pp. 313-344.

Marcus, Richard, Misgivings about american exceptionalism: Court access as a zero-sum game, en Uzelac, Alan - VAn Rhee, C. H. (eds.), Revisiting procedural buman rights: Fundamentals of civil procedure and the changing face of civil justice (Cambridge, Intersentia Metro, 2017), pp. 53-75.

Матsuмото, Hiroyuki, The reception and transmission of the law of civil procedure in Japan. The experience in Japan, en Deguchi, Masahisa - STORME, Marcel (eds.), The reception and transmission of civil procedural law in the global society. Legislative and legal educational assistance to other countries in procedural law (IAPL, Maklu Antwerpen - Apeldoorn, Maklu Publishers, 2008), pp. 137-148.

Morgan, Edmund M., The jury and the exclusionary rules of evidence, en The University of Chicago Law Review, 4/2 (1937), pp. 247-258.

Neuberger, David, Docketing: Completing case management's unfinished revolution, en Ninth Lecture in Implementation Programme Solicitors' Costs Conference (London, 2012), disponible en World Wide Web: https://www.judiciary.gov.uk/wp-content/uploads/ JCO/Documents/Speeches/mor-speech-solicitors-cost-conference-lecturefeb2012.pdf (consulta, 13 de febrero de 2018).

— Framing a new procedural culture, en Civil Justice Quaterly, 34/3 (2015), 237-243.

NGWAsIRI, C. N., The role of the judge in french civil proceedings, en Civil Justice Quarterly, 9 (1990), pp. 167-173.

Nokes, G. D., The English jury and the law of evidence, en Tulane Law Review, 31 (19561957), pp. 153-172.

O’Hare, John; Browne, Kevin, Civil litigation (17th ed., London, Sweet \& Maxwell, 2015).

Oberhammer, P., Domej, T., Improving the efficiency of civil justice: Some remarks from an Austrian perspective, en VAN RHeE, C.H. - Uzelac, Alan (eds.), Civil justice between efficiency and quality: From ius commune to the Cepej (Intesentia Metro, 2008), pp. 61-70.

OTEIzA, Eduardo, Civil procedure reforms in Latin America: The role of the judge and the parties in seeking a fair solution, en The Supreme Court Review, 49 (2010), pp. 225-246.

PRÜTTING, Hans, International sources of german civil procedure, en Deguchi, Masahisa STORME, Marcel (eds.), The reception and transmission of civil procedural law in the global society. Legislative and legal educational assistance to other countries in procedural law (IAPL, Maklu Antwerpen - Apeldoorn, Maklu Publishers, 2008), pp. 249-260.

Slapper, Gary - KelLy, David, The English legal system (Twelfth ed., Routledge, 2011). SorabjI, John, Austerity's effect on English civil justice, en Erasmus Law Review, 8/4 (2015), pp. 159-173.

- English civil justice after Woolf and Jackson reform: A critical analysis (London, Cambridge University Press, 2014).

- The road to new street station: Fact, fiction and the overriding objective, en European Business Law Review, 23/1 (2012), pp. 77-89.

- Managing claims (Tianjin Conference, IAPL, 2017).

Soulez Lariviere, Daniel, Overview of the problems of french civilprocedure, en The American Journal of Comparative Law, 45 (1997), pp. 737-746. 
STURNER, Rolf, Some European remarks on a new joint project of the American Law Institute and UNIDROIT, en The International Lawyer, 34/3 (2000), pp. 1071-1086.

Sward, Ellen E., Values, ideology and the evolution of the adversary system, en Indiana Law Journal, 64/2 (1989), pp. 301-355.

TARUFFO, Michele, Una alternativa a las alternativas: Modelos de resolución de conflictos, en Páginas sobre justicia civil (Madrid, Marcial Pons, 2009)

Tulibacka, Magdalena, The ethos of the Woolf reforms in the transformations of post-socialist civil procedures: Case study of Poland, en DWYER, Déirdre (ed.), The civil procedure rules ten years on (Oxford, Oxford University Press, 2012), pp. 395-413.

Uzelac, Alan, Croatia: Omnipotent judges as the cause of procedural inefficiency and impotence, en Van Rhee, C. H. - Yulin, Fu (eds.), Civil litigation in China and Europe: Essays on the role of the Judge and the Parties (Dordrecht, Springer, 2014), pp. 197-221

Van Caenegem, R.C., An historical introduction to private law (trad. de Johnston, D.E.L., Cambridge University, 1992).

Van Rhee, C. H., Verkerk, R., Civilprocedure, en Smits, Jan M. (ed.), Elgar encyclopedia of comparative law (Second ed., Edward Elgar Publishing Limited, 2012), pp. 120-134.

Van Rhee, C. H., The influence of the french code de procédure civile (1806) in 19th century Europe, en CADIET, L. - CANIVET, G. (eds.), De la commémoration d'un code à l'autre: 200 ans de procédure civile en France (Paris, LexisNexis/Litec, 2006), pp. 129-165.

- Towards a procedural ius commune?, en Smits, J. - Lubbe, G. (eds.), Remedies in ZuidAfrika en Europa (Antwerpen, 2003), pp. 217-232.

- European traditions in civil procedure, Van RHEE, C. H. (ed.), (Intersentia Antwerpen - Oxford, 2005).

- The development of civil procedural law in twentieth-century Europe, en Russian Yearbook on Civil Procedure \& Arbitration, 7-8 (2011), pp. 11-25.

- The development of civil procedural law in twentieth century Europe: From party autonomy to judicial case management and efficiency, VAN RHEE, C. H. (ed.), Judicial case management and efficiency in civil litigation (Intersentia Antwerp - Oxford - Portland, 2008).

- European Traditions in civil procedure, (Intersentia Antwerpen - Oxford, 2005).

VORRASI, Kenneth M., England's reform to alleviate the problems of civil process: A comparison of judicial case management in England and the United States, en Journal of Legislation, 30/2 (2004), pp. 361-387.

Wall, Andrea, Austria \& Germany: A bistory of successful reforms, en VAN RHEe, C.H. Yulin, Fu (eds.), Civil litigation in China and Europe: Essays on the role of the judge and the parties (Dordrecht, Springer, 2014), pp 141-177.

Wijffels, Alan, France: Powers of the judge, en VAn RheE, C. H. (ed.), Europeans traditions in civil procedure (Intersentia Antwerpen - Oxford, 2005), pp. 269-280.

- French civil procedure (1806-1975), en Van RheE, C. H. (ed.), Europeans traditions in civil procedure (Intersentia Antwerpen - Oxford, 2005), pp. 25-47.

- The code de procédure civile (1806) in France, Belgium and the Netherlands, en VAN RHEE C. H.; Heirbaut, D.; STORMe, M. (eds.), The french code of civil procedure (1806) after 200 years: The civil procedure tradition in France and abroad (Kluwer, 2008), pp. 5-73.

Woolf, Harry, Civil justice in the United Kingdom, en The American Journal of Comparative Law, 45/4 (1997), pp.709-736.

- Access to Justice, Interim Report. disponible en World Wide Web: http:/ / webarchive. nationalarchives.gov.uk/+/http://www.dca.gov.uk/civil/interim/chap3.htm (consulta, 25 de febrero de 2018).

- Access to Justice: Final report to the Lord Chancellor on the civil justice system in England and Wales (1996) Final report, disponible en World Wide Web: http:/ /webarchive. 
nationalarchives.gov.uk/+/http:/www.dca.gov.uk/civil/final/contents.htm (consulta, 24 de febrero de 2018).

Zuckerman, A. A. S., Cranston, Ross (eds.), Reform of civil procedure: Essays on "acess to justice" (Oxford University Press, 1996).

ZuCKerman, Adrian A.S., Justice in crisis; comparative dimensions of civil procedure, en ZucKERMAN, Adrian A.S. (ed.), Civil justice in crisis, comparative perspectives of civil procedure (Oxford, Oxford University Press, 1999).

- Court adjudication of civil disputes: A public service that needs to be delivered with proportionate resources, within a reasonable time and at reasonable cost, disponible en World Wide Web: http://www.aija.org.au/ac06/Zuckerman.pdf, p. 8, (consulta, 18 de febrero de 2015).

- Editor's Note. Must a fraudulent litigant be allowed to think: if the fraud is successful, I will gain much; if it is not, I will still recover my legitimate claim?, en Civil Justice Quaterly, 30/1 (2011), pp. 1-14.

- Conference on "The ALI-UNIDROIT principles and rules of transnational civil procedure", hosted by the British Institute of International and Comparative Law, London May 24, 2002, en Civil Justice Quarterly, (2002), pp. 322-325

— The revised CPR 3.9: a coded message demanding articulation, en Zuckerman, A.A.S. (ed.), Civil Justice Quarterly, Special Issue: The implementation of Sir Rupert Jackson's review of civil litigation costs, 32/2 (2013), pp. 123-138 